\title{
The connections and contradictions in student responses to contextualised tasks
}

Diane Dalby

\section{School of Education, University of Nottingham, UK}

The development of skills that enable adults to apply mathematics in life and work is a recurring theme in mathematics education, but it requires a transferability of learning that cannot be assumed. Variations in performance between formal and informal learning situations (Lave, 1988; Nunes, Schliemann, \& Carraher, 1993) provide evidence that learning is not automatically transferred, and observations of mathematics in work situations (Williams \& Wake, 2007) illustrate how mathematics in 'real life' rarely resembles classroom mathematics. The unseen boundaries are only too apparent to students who often fail to see the relevance of classroom mathematics to their lives and lose motivation to engage with the subject. Exposing students to a range of applications of mathematics in genuinely authentic contexts may provide a means of developing transferability (Boaler, 1998) but students' interpretations of such tasks can vary. Understanding students' responses to contextualised tasks and their perceptions of relevance is important if contexts are to be used effectively to engage students in developing transferable skills for the future.

This study examines the responses of vocational students in three Further Education colleges to a range of contextualised tasks. It provides some insight into their perceptions of the relevance of a these 'realistic' tasks to their lives. Seventeen small focus groups of students from the vocational areas of Construction, Public Services and Hair and Beauty were each shown three unfamiliar contextualised functional mathematics tasks from a bank of ten. Each task involved the application of mathematics to a situation that might hold some connection to the students' vocational area or personal lives. The focus groups were asked about their first impressions of the task, whether it was relevant to them in any way and whether they would learn any useful skills through doing the task. The discussions were recorded, transcribed and coded to identify the main themes.

Firstly, students made connections at three different levels in their attempts to relate to the tasks.

- Superficial level (recognition): students recognised isolated words that had connections to their lives such as hair dyes (hairdressing) or a sink unit (plumbing) and used these to make conclusions about relevance without considering the actual content of the task.

- Scenario level (recall): students recalled a similar situation within their personal experience and identified with the scenario but overlooked differences in the mathematical task involved.

- Task level (resemblance): students identified the task as authentic and relevant because it closely matched a 'real life' task they had experienced or expected to perform in the future.

Secondly, distinctions were made between tasks that had an immediacy of usefulness and those that might be of value to them in the future. Those with immediate practical use were perceived to be most relevant whilst those they only expected to be encounter later were deemed less relevant.

Thirdly the context did stimulate a type of interest which affected their judgements regarding relevance. Generally, students related most strongly to situations they could identify as part of their vocational specialism, although connections to their personal lives also generated interest.

The results of this study suggest that these three 'dimensions' (connection, immediacy of usefulness and interest) could provide a useful conceptual framework for understanding students' perceptions of relevance. However, the connections made by students were easily fractured by apparent 
contradictions between their personal experience and the scenario presented. The limitations of individual personal experience meant some students failed to see the relevance of a task because the scenario was, as yet, unfamiliar. Students were also sensitive to inconsistencies in details. For example, a reference to tubes of hair dye was consistent with salon practice for most students but some students who only used dispensers for hair dye rejected the task as unrealistic and irrelevant.

For these students the context acted as a potential source of interest that linked the classroom to their lives. A relevant context motivated students to engage with the task and facilitated understanding, but contexts could also confuse and the connections to their lives were often fragile. Further study into how teacher mediation influences these connections and contradictions in the classroom may serve to extend this initial insight into the relevance of contextualised tasks and their value in the learning process.

\section{Acknowledgments}

This research is funded by a studentship from the University of Nottingham.

\section{References}

Boaler, J. (1998). Open and closed mathematics: Student experiences and understandings. Journal for Research in Mathematics Education, 29(1), 41-62.

Lave, J. (1988). Cognition in practice: Mind, mathematics, and culture in everyday life. New York: Cambridge University Press.

Nunes, T., Schliemann, A.D., \& Carraher, D.W. (1993). Street mathematics and school mathematics. Cambridge: Cambridge University Press.

Williams, J., \& Wake, G. (2007). Black boxes in workplace mathematics. Educational Studies in Mathematics, 64(3), 317-343. 